\title{
Braking Principle and Control Technology of Automobile ABS System
}

\author{
Yuanpeng Zhu
}

(School of automotive engineering, Wuhan University of Technology, Wuhan, Hubei, 430070)

Keywords: automobile; ABS control; working principle; braking performance

\begin{abstract}
This paper introduces the composition of the anti-lock braking system and the braking principle of the wheel and the whole vehicle. It lists the control method and work process of the wheel and the vehicle. The processing method of the car motion parameter is also discussed. In addition, combining the different situations, the analysis of the car under extreme conditions of control ideas for the future development of ABS technology has a certain reference value.
\end{abstract}

\section{Introduction}

ABS (Anti-lock Braking System) is to prevent the car in the process of emergency braking tire lock developed by the car electronic control safety devices, the main idea is the original car braking system based on an increase in the vehicle Electronic control equipment. Although the manufacturers of the ABS system composed of different structures, but in general by the sensor on the hardware, electronic control unit (ECU) and brake pressure regulator composed of three parts. It can adjust the slip rate as the main goal, in the process of braking the car according to the friction between tires and road conditions, automatically adjust the wheel braking torque to achieve maximum braking performance, thereby reducing the braking distance and the car Braking time, reduce tire wear, extend the life of the car. At the same time, ABS system can effectively reduce the front wheel locking, loss of turning ability or due to the rear wheel lock and the possibility of accidents such as drift, driving for the safety and stability of the vehicle provided a guarantee. Therefore, the current ABS has become a standard configuration of the mainstream car.

ABS technology began in 1932, the first from the UK patent non-slip UK382241. Germany Robert-Bosch Company in 1936 will be used to measure the speed of the electromagnetic sensor to join them and apply for a patent in 1978 with Buzz developed a digital microprocessor controller with four rounds of ABS, and first began to apply to the Mercedes-Benz Cars, laid the basic model of ABS. In the late 80's, as the technology matures, many auto parts companies began to produce ABS products, the technology is also popular among civil vehicles.

\section{ABS working principle}

The motion state of the wheel is divided into pure rolling, side rolling sliding and pure sliding. The wheel center movement speed is determined by comparing with the wheel rolling speed. The wheel speed is greater than the wheel circumference rolling speed and the braking wheel does not occur when the brake locking, the edge of the wheel rolling edge sliding movement, brake wheel braking force at this time, the circumferential rolling speed gradually decreased, and body inertia factors Will continue to move forward, between the tire and the road surface slip phenomenon. When the circular rolling speed gradually decreased to 0 , the brake wheel is completely locked, the body just rely on inertia forward movement, when the wheel for pure sliding movement.

Under normal circumstances, according to the wheel rolling speed and the center of the central speed, you can get the wheel slip rate, the performance of the wheel in the process of sliding the degree of the brake, the definition is:

Where: S--slip ratio

--wheel angular velocity

$\mathrm{R}$--wheel radius

V--vehicle operating speed 
Under normal circumstances, the commonly used slip rate to indicate the movement of the tire state; common friction coefficient to indicate the tire road conditions. Through a large number of experiments, the relationship between tire longitudinal friction coefficient and slip rate is obtained under different road conditions. As shown in Fig. 1, in a certain range, the longitudinal friction coefficient is positively related to the slip ratio of the tire. When the coefficient reaches the maximum, there is a negative correlation between them. When the longitudinal friction coefficient reaches the maximum value, the corresponding slip ratio is called the optimum slip rate, and the optimum slip rate of different road surface is different.

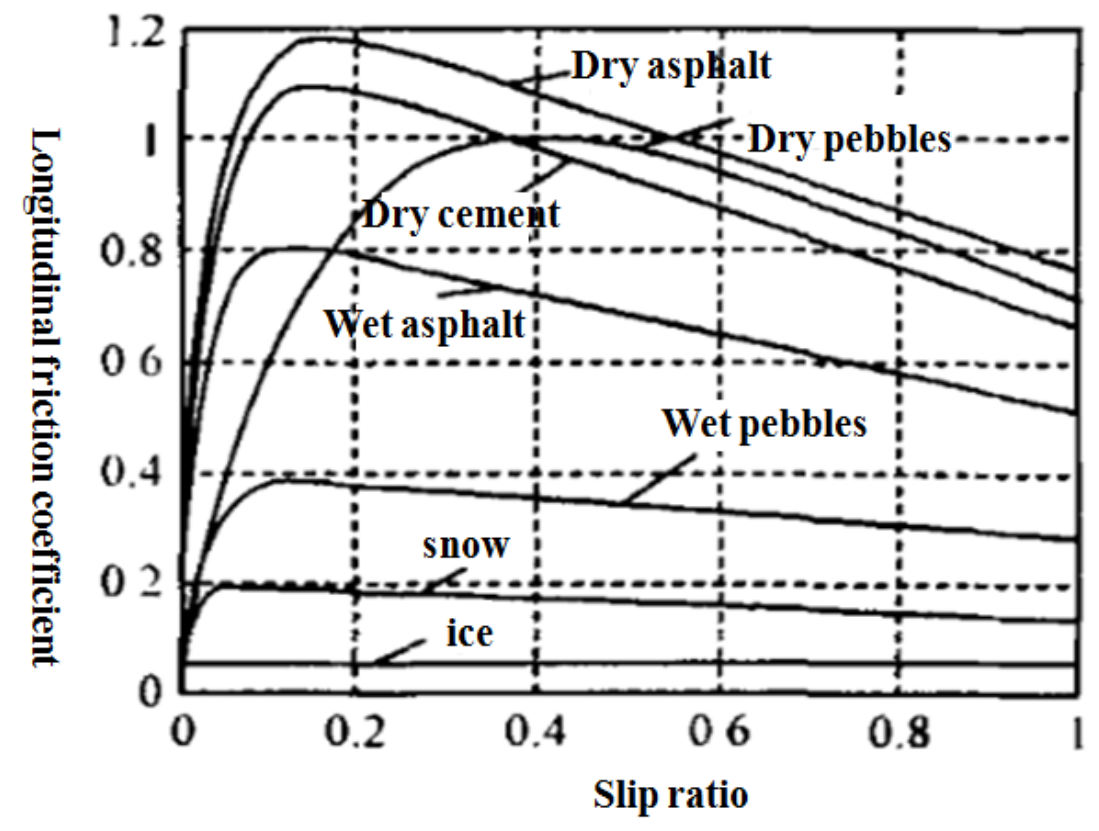

Fig. 1 The relationship between longitudinal friction coefficient and slip rate under different road conditions

At the same time, according to the previous discussion, we can know that when the wheel to pure rolling motion, the slip rate of 0 ; when the vehicle for pure sliding, slip rate of 1 . The ABS brake principle is to slip rate control in the ideal range, in order to obtain the best braking performance. Sensor in the car brake process, the monitoring of wheel angular velocity and longitudinal acceleration of the car body, and as an input signal, calculate and analyze the instant slip rate. Where the wheel angular velocity can be obtained directly with an electromagnetic or photoelectric wheel speed sensor; the wheel speed can be obtained from the angular velocity and the acceleration of the wheel for the reference speed of the vehicle. And then the braking torque is adjusted by the control command for boosting, holding or decompressing the brake pressure based on the difference between the initially set reference slip rate and the resultant slip ratio, and then, based on the braking torque and The obtained slip rate is used as the reference slip rate to adjust the braking torque again. The closed-loop cycle keeps the wheels in the optimum braking state, the longitudinal friction force of the tire is the largest, and the braking effect of the vehicle is the best.

\section{Tire control method for ABS}

As the car in the process of driving too many factors, so in the course of the study, the use of separation method will be car decomposition of the body mass concentration of a center of mass and unsprung two parts. Based on the simple model, the nature of ABS control technology is studied. At present, the common ABS control technology is divided into two kinds: one is based on slip rate of the anti-lock control method; one is based on the wheel acceleration logic threshold control method.

\subsection{Fuzzy Control Method}

Fuzzy control is a simulation of human thinking, which is a kind of computer numerical control 
technology based on fuzzy set theory, fuzzy linguistic variables and fuzzy logic reasoning, which has good robustness and flexibility of control rules. But the downside is that, in the face of different roads and models, the proportion of its controller parameters need to be re-adjusted, and the adjustment process is very dependent on the test by trial and Minato get the data.

The control process is roughly as Fig.2: the brake pedal through the pedaling force output signal, and the signal input to the braking system corresponds to a pre-set brake pressure, while with the output from the sensor variables into the calculation module. After the operation, the actual target pressure value is output to the braking system. And then through the air pressure system to obtain the target vehicle braking torque, and brake operation. At the same time the detection device to calculate the actual slip rate of the car, the input to the fuzzy controller, the actual slip rate will be set in the desired slip rate error, through the fuzzy control algorithm to calculate the control pressure feedback to the braking system, get a good braking effect and handling stability.

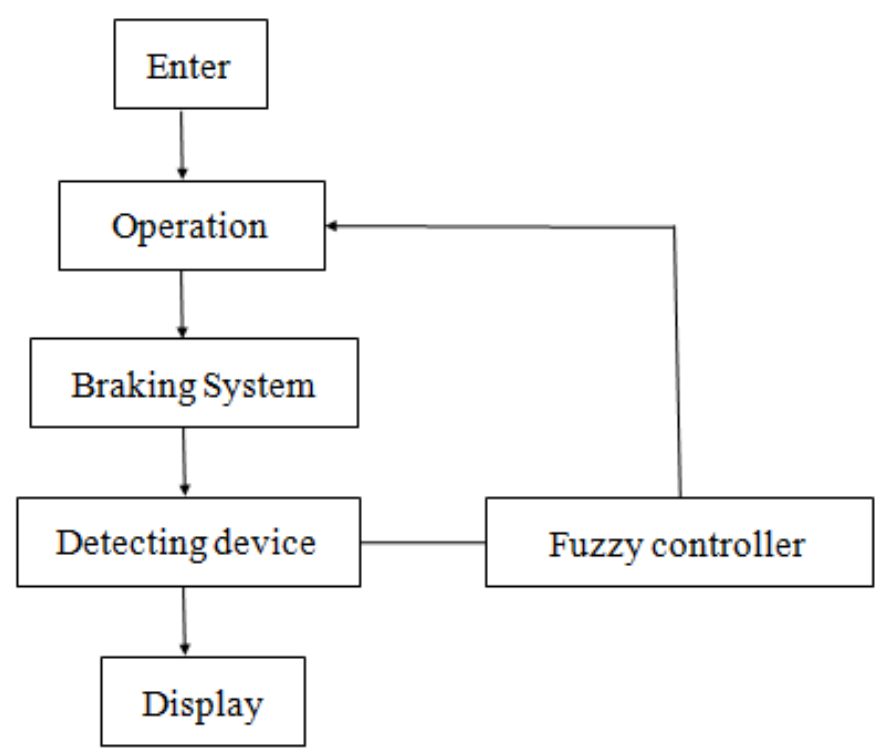

Fig.2 Fuzzy control process

\subsection{Logic Threshold Control Method}

In the case of ignoring the air resistance, usually used to establish the model of the method of movement, said the movement of vehicles and wheels and force conditions.

Among them, $\mathrm{M}$ is the vehicle quality; $\mathrm{R}$ is the wheel radius; $\omega$ wheel angular velocity; $\mathrm{FN}$ is the wheel friction; F $\omega$ is the wheel rolling resistance; $\mathrm{J}$ is the wheel inertia; Tb is the braking torque; $\mathrm{T}$ is the braking torque; is the road adhesion coefficient; $\mathrm{N}$ for the wheel and the ground normal reaction force; $\mathrm{J} \omega$ ' that the angular momentum of the wheel. When the braking torque Tb less than the braking torque T, J $\omega$ ' is less than zero, the car is in a deceleration state; the other hand, the car is in the accelerated state. According to the above principles can be set up two appropriate wheel acceleration and deceleration threshold to achieve the control of brake pressure to prevent the wheel lock occurs. In addition, if only the wheel acceleration and deceleration is used as a reference, it is possible to disable the anti-lock control in the case of initial and high-speed emergency braking. Therefore, the logic threshold method also needs to join the slip rate, with the wheel acceleration and deceleration in combination, in different road surface brake control.

\subsection{For the acquisition signal processing}

As the wheel speed measurement process, will be machining error, electromagnetic interference, vehicle vibration and other factors, resulting in the measurement process will be relatively large fluctuations, it may accidentally trigger the ABS system, resulting in the driver's operation failure. Therefore, it is necessary to further smoothing the data collected to ensure the accuracy of the signal, and generally through the digital filtering technology to complete.The digital filtering research above, the commonly used methods to filter gain constant exponential smoothing data filtering 
technology, in addition to complex filtering technology such as Kalman filter technology in wheel speed signal processing applications, in addition to the car for data detection Real-time requirements are relatively high, the filter can not be too complex in general, the amount of computing should be small.

\subsection{Synchronous control of front wheel of automobile}

In the actual test, ABS work often occur when the phenomenon of vehicle deviation, while the direction of the car driving process is mainly determined by the two front wheels, it can be learned that the main reason is due to the existence between the front wheel by the system Dynamic pressure caused by the difference between the speed difference, so in the control process should ensure that the two are in sync between the state and reduce the pressure difference between each other. Therefore, the following conclusions can be drawn from the analysis: First, when one of the front wheels into the decompression state, the other front wheel should be in a state of pressure or decompression, specifically by the steering wheel is deflected and the initial car set parameters To determine, to prevent the pressure difference between the two rounds is too large. Second, two front wheels if one is in the supercharged state, the other must be in a pressurized state, to prevent the car in the deceleration process, suddenly change the direction of the accident caused by the road.

\section{Vehicle control method of ABS}

As the car in the process of moving very complex, will be affected by the road conditions, such as the impact of wind and so on, resulting in lateral car torque, resulting in steering failure of the accident. Therefore, if only consider the tire braking situation is far from enough, should also be the vehicle as a control object, so that the movement of the deviation as small as possible, and even to ensure that the tire suffered lateral force and longitudinal force, sacrifice part The slip rate of the vehicle.

\subsection{EBD control}

In order to prevent the rear wheel locking due to the car side slip or even drift phenomenon, usually using EBD control method to solve. EBD uses electronic technology to replace the traditional proportional valve to control the vehicle hydraulic braking system before and after the bridge braking force distribution, the basic idea is: first as much as possible to increase the rear wheel braking force, and by the sensor to monitor, The brake pressure is reduced by the hydraulic brake immediately, which is characterized by high adjustment frequency, small adjustment range and accurate control, so that the rear wheel can obtain the maximum braking force under the circumstance of ensuring that the rear wheel is first locked.

\subsection{Steering Dynamics Control}

In the actual road, the vehicle yaw moment due to the sudden changes in its steering characteristics, if the ABS is still the wheel slip rate control in the vicinity of the best slip rate, the vehicle to obtain the yaw balance torque is very limited. So in some extreme circumstances, you can make a tire locking at the expense of, to get the maximum balance of the yaw moment. Taking the longitudinal velocity and yaw rate of the car as parameters, the feedback control method is adopted to control the yaw and transverse speed of the vehicle. This method can correct the yaw rate of the vehicle more quickly than the yaw moment control, and the slip ratio is kept constant, and improves the lateral performance of the vehicle. 


\section{Conclusion}

In summary, automotive ABS technology has very important significance for the car, he can greatly enhance the braking performance of the car to improve vehicle safety. At the same time, ABS system not is only need to prevent wheel lock, for the movement of the vehicle should also be adjusted to avoid the extreme conditions due to yaw moment to the car's steering system failure. With the development of electronic technology, the detection and calculation of the parameters of the car will be greatly improved, which will further promote the development of ABS technology, the car has a higher security.

\section{References}

[1] T.W. Zheng, F.F. MA.Research on control method of logic gate of automobile ABS [J].Journal of Chongqing University of Posts and Telecommunications (Natural Science Edition), 2011,23 (4) [2] Z.W. Lin, S.R. Yan.Automobile ABS control technology and development [J].Journal of Fujian Institute of Technology, 2008 (6)

[3] W.T. Han, M.Q. Liu, Z.Y. Yuan.Study on fuzzy control method of automobile ABS [J].Highway and Qiyun, 2004 (1)

[4] L. Sun.The Development and Technical Trend of Automobile ABS [J].Equipment Manufacturing Technology, 2009 (7)

[5] R. Fan, X.T. Zhang.Study on ABS Control Method of Automobile Based on ARM CORTEX-M4 Technology [J] .2013 (11) 